\title{
Mediastinal lymphangioma in an adult
}

\author{
Mouhamad Nasser, MD, ${ }^{\mathrm{a}}$ Kais Ahmad, MD, ${ }^{\mathrm{b}}$ and Vincent Cottin, MD, PhD, ${ }^{\mathrm{a}, \mathrm{b}}$ Lyon, France
}

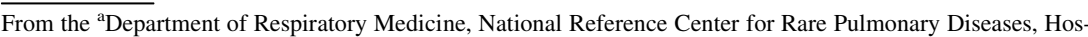 \\ pices Civils de Lyon, Lyon, France; and ${ }^{\mathrm{b}}$ Claude Bernard Lyon 1 University, University of Lyon, INRA, \\ UMR754, Lyon, France. \\ Funding: There was no funding for this article. \\ Disclosures: Authors have nothing to disclose with regard to commercial support. \\ Received for publication Oct 31, 2017; revisions received Dec 14, 2017; accepted for publication Dec 23, 2017; \\ available ahead of print Feb 2, 2018. \\ Address for reprints: Vincent Cottin, MD, PhD, Service de Pneumologie, Hôpital Louis Pradel, 28 Ave Doyen \\ Lepine, Lyon Cedex F-69677, France (E-mail: vincent.cottin@chu-lyon.fr). \\ J Thorac Cardiovasc Surg 2018; 155:e195-7 \\ $0022-5223 / \$ 36.00$ \\ Copyright (C) 2018 by The American Association for Thoracic Surgery \\ https://doi.org/10.1016/j.jtcvs.2017.12.119
}

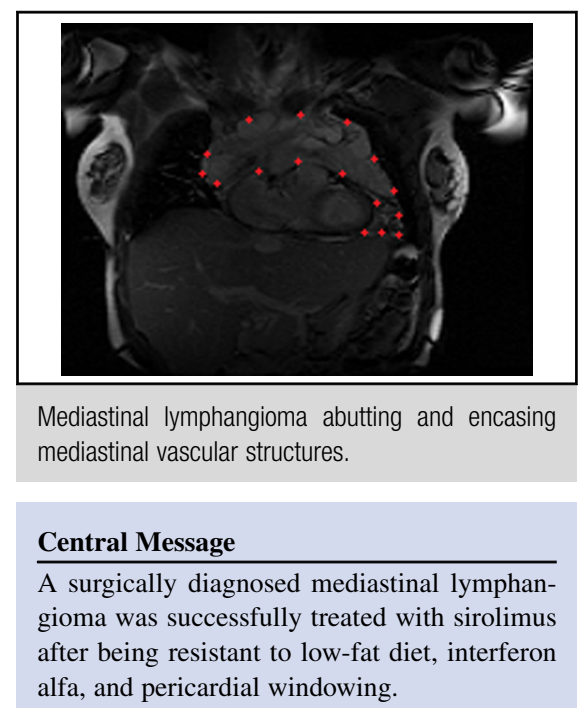

See Editorial Commentary page e199.
Lymphangiomas are hamartomatous congenital lymphatic malformations involving primarily skin and subcutaneous tissue. More than $90 \%$ of cases occur in children younger than 2 years; in these cases, the fetal neck (75\%) and axilla $(20 \%)$ are most commonly involved. In adults, all lymphatic chain territories may be involved; however, thoracic lymphangiomas are both the most common and the most severe. Lymphangiomas are classified according to their histologic features as simple, cavernous, or cystic. Mediastinal lymphangioma is a very rare mediastinal tumor in adults ( $<5 \%$ of lymphangiomas are mediastinal). In most cases, diagnosis is delayed until surgical exploration. Although the majority of these tumors remain clinically silent, others progress locally and can jeopardize the patient's prognosis. ${ }^{1}$ To date, surgery remains the standard of care in reported cases for patients with progressive symptoms. ${ }^{2}$ Although data regarding other treatment modalities are sparse, chemotherapy, radiotherapy, and laser therapy have been reported, with highly variable efficacy. ${ }^{3}$ Because of the underlying histopathology of mediastinal lymphangioma, mechanistic target of rapamycin (mTOR) inhibitors seem a plausible alternative in impeding lymphangiogenesis. Because mTOR inhibitors have been used successfully in the pediatric population with lymphangioma-related diseases, ${ }^{3-5}$ their use would seem advisable in the treatment of adult patients with similar disease morphology. Although the role of sirolimus has been described in children, however, its effect on mediastinal lymphangioma in adults has not been reported. We present here the first case of mediastinal lymphangioma in an adult successfully treated with sirolimus without the need for surgical intervention.

\section{CASE REPORT}

A 32-year-old woman presented to the emergency department with subacute onset of fever, shortness of breath, and fatigue. Physical examination revealed hemodynamic instability, distended jugular veins, and increased liver span, with no edema of the lower limbs. Echocardiography showed a 20- to 40-mm thick circumferential pericardial effusion with diastolic dysfunction. Decompensated cardiac tamponade was diagnosed, and the patient underwent surgical creation of a pericardial window. Postoperative magnetic resonance imaging demonstrated soft tissue infiltration of the mediastinum, with hypersignal on $\mathrm{T} 2$ and isosignal on $\mathrm{T} 1$, encasing the aortopulmonary window (Figure 1, A) and the subcarinal space and abutting the posterior mediastinum without vascular invasion (Figure $1, B$ and $C$ ). This lesion was measured at $130 \mathrm{~mm} \times 118 \mathrm{~mm}$, with the presence of a rim of pericardial effusion anterior to the right heart cavities. Bilateral pleural effusions were also present. Surgical biopsy of the mediastinum demonstrated adipose tissue partially infiltrated with lymphatic vessels paved with endothelial cells. The septa were thin and infiltrated mainly with lymphocytes. Some smooth 

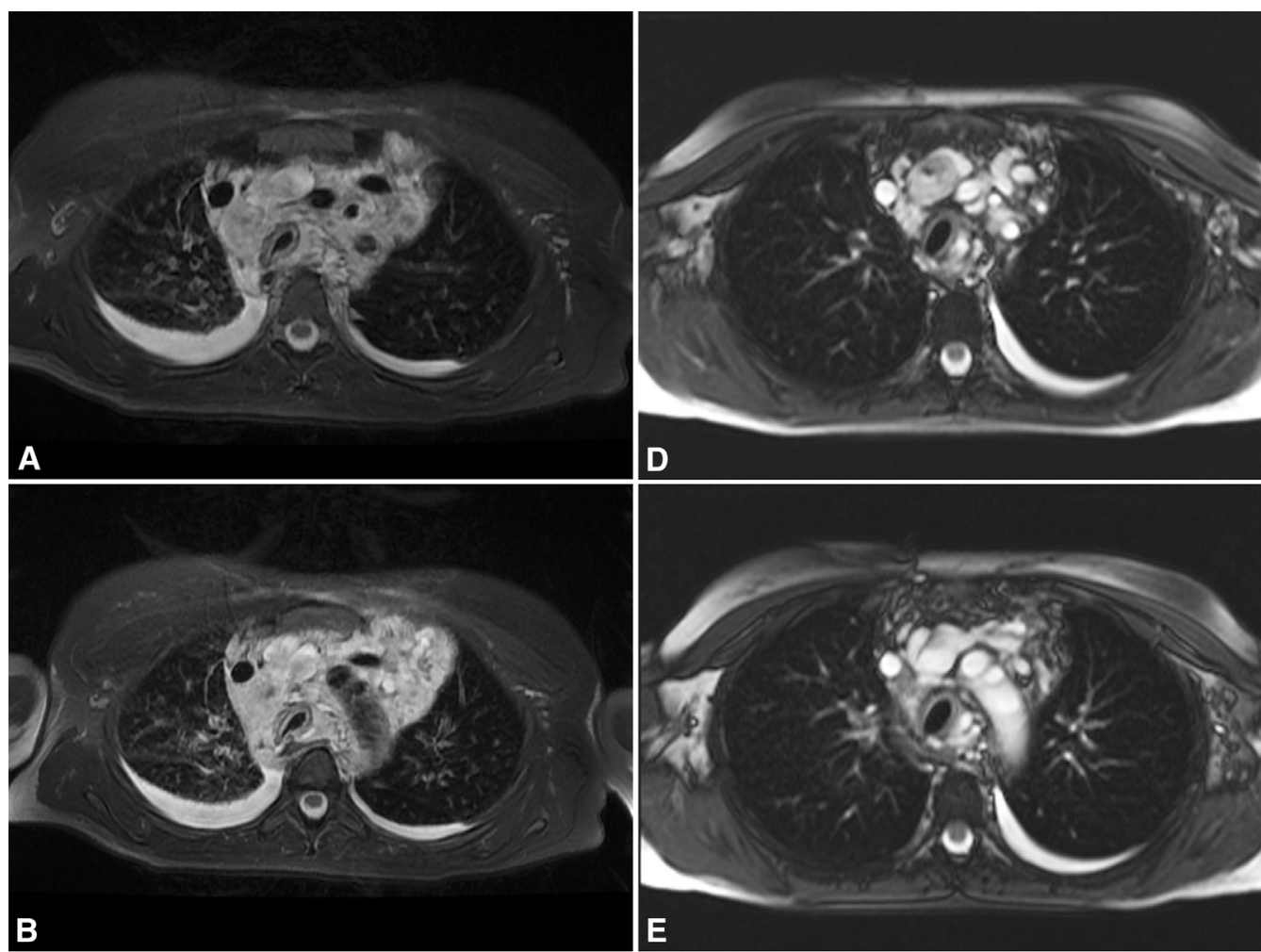

D
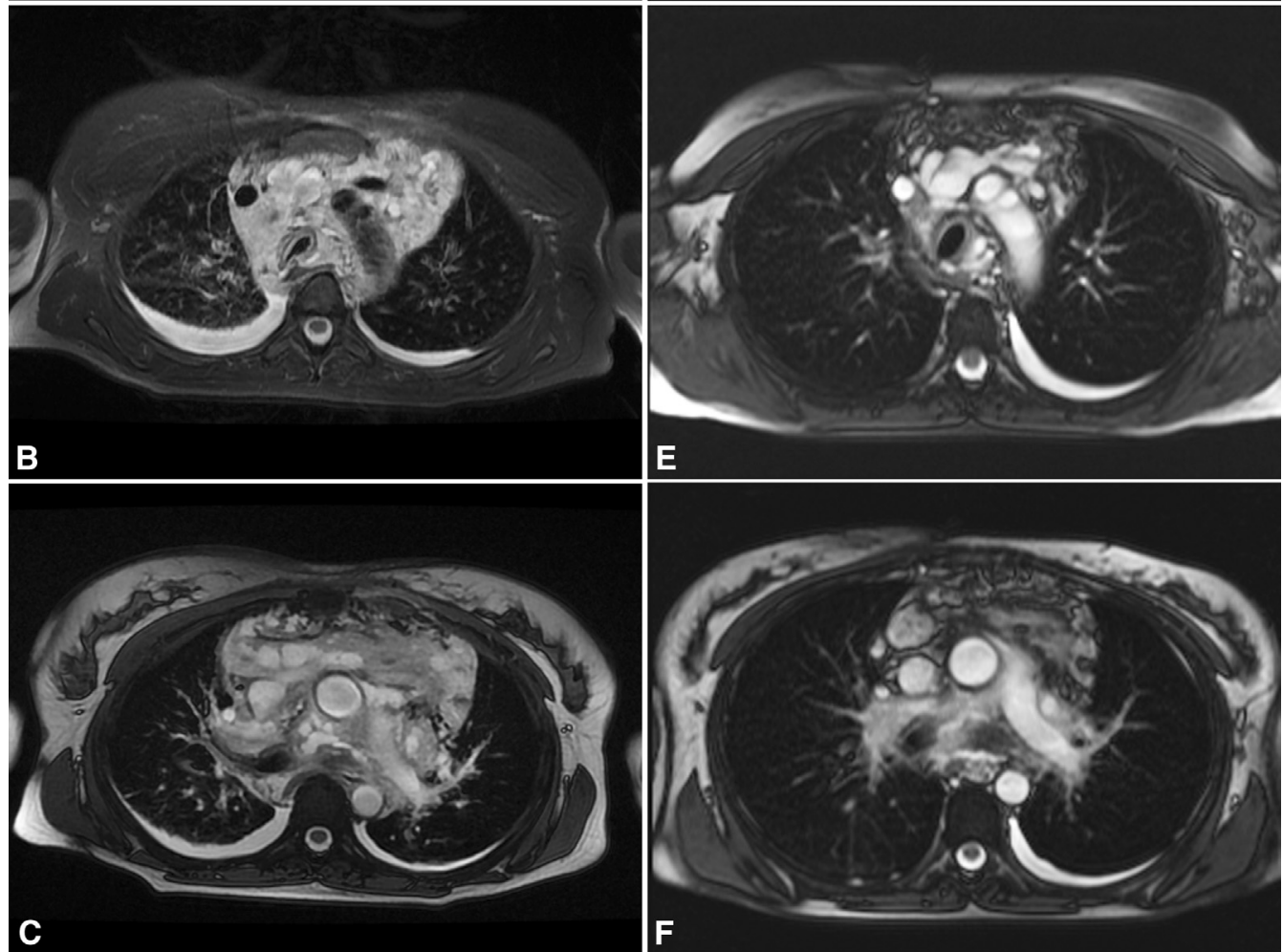

FIGURE 1. A, B, and C, Magnetic resonance imaging of the mediastinum before sirolimus treatment shows mediastinal infiltration with encasement of the ascending aorta and bilateral pleural effusions. D, E, and F, Magnetic resonance imaging 10 months after sirolimus shows marked reduction of mediastinal lymphangioma size, with complete resolution of the right pleural effusion.

muscle cell tatters were observed. Immunohistochemical testing results were positive for desmin, actinmuscle1A4, and CD31 and were negative for HMB 45. These findings were highly suggestive of mediastinal lymphangioma.

During the subsequent 3 years, the patient was admitted to the hospital multiple times for shortness of breath, intractable cough with foamy sputum, and abundant recurrent pleural effusions. Several medical treatments were unsuccessful, including inhaled corticosteroids, subcutaneous interferon alfa for 8 months, and low-fat diet enriched with medium-chain triglycerides for 28 months (Video 1). After these treatment failures, written, informed consent was obtained for off-label treatment with the mTOR inhibitor sirolimus, which was initiated at $2 \mathrm{mg} / \mathrm{d}$, with a target trough serum level of 5 to $15 \mathrm{mg} / \mathrm{L}$. Dramatic clinical improvement was obtained within 10 months, with complete and sustained remission of the mediastinal mass at imaging and disappearance of the right pleural effusion (Figure 1, $D-F)$, obviating the need for surgery. Sirolimus treatment was well tolerated for 38 months, until subacute sirolimus-induced pneumonitis occurred and the drug was 


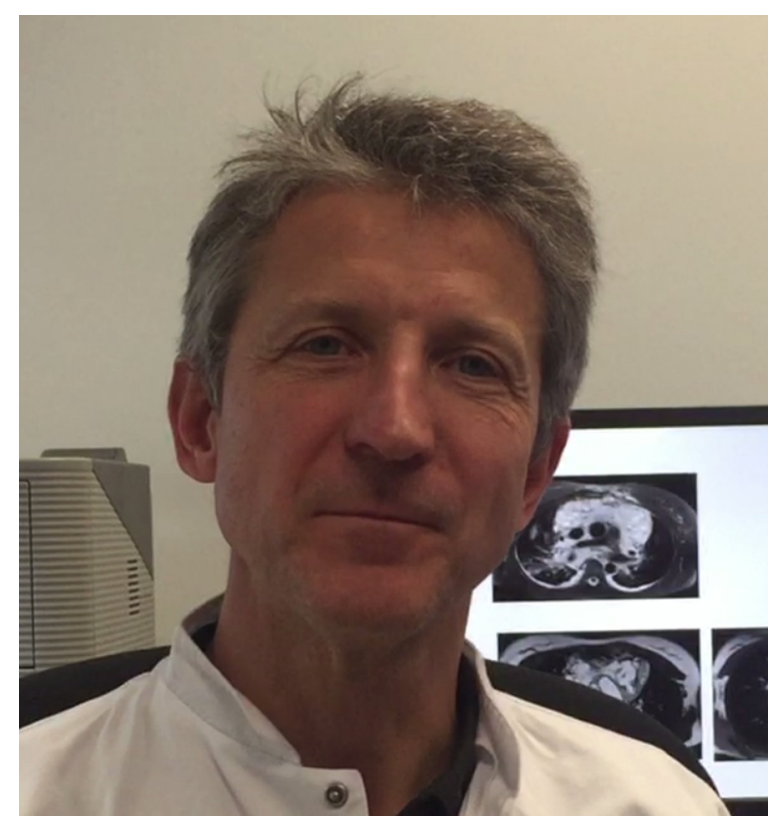

VIDEO 1. A 2-minute video shows one of the authors (V.C.) summarizing the case and highlighting relevant points related to the patient's diagnosis and management. Video available at: http://www.jtcvsonline.org/article/ S0022-5223(18)30038-2/fulltext. discontinued. This treatment course resulted in complete resolution of the bilateral lung infiltrates.

\section{DISCUSSION}

To the best of our knowledge, this is the first case report on the use of mTOR inhibition to treat an adult with mediastinal lymphangioma. Our case points to the beneficial effect of mTOR inhibitors (in this case sirolimus) in the management of such patients. Interestingly, the patient had a stable mediastinal disease course 6 months after drug discontinuation. This finding highlights a probable curative effect rather than a suppressive effect and is deserving of further investigation.

\section{References}

1. Shenoy SS, Barua NR, Patel AR, Culver GJ, Jennings EC. Mediastinal lymphangioma. J Surg Oncol. 1978;10:523-8.

2. Fokkema JP, Paul MA, Vrouenraets BC. Mediastinal lymphangioma in an adult Ann R Coll Surg Engl. 2014;96:e24-5.

3. Bagrodia N, Defnet AM, Kandel JJ. Management of lymphatic malformations in children. Curr Opin Pediatr. 2015;27:356-63.

4. Akyüz C, Ataş E, Varan A. Treatment of a tongue lymphangioma with sirolimus after failure of surgical resection and propranolol. Pediatr Blood Cancer. 2014;61:931-2.

5. Huber S, Bruns CJ, Schmid G, Hermann PC, Conrad C, Niess H, et al. Inhibition of the mammalian target of rapamycin impedes lymphangiogenesis. Kidney Int. 2007;71:771-7. 